\title{
Performance and Operational Status of Muon Detectors in the Telescope Array Experiment
}

\author{
T. Nonaka ${ }^{* a}$, R. Cady $^{b}$,D. Ivanov ${ }^{b}$, J.N. Matthews ${ }^{b}$, S. Ogio ${ }^{f}$, T. Okuda ${ }^{e}$, H. Sagawa ${ }^{a}$, \\ N. Sakurai ${ }^{f}$, B.T. Stokes ${ }^{b}$, and R. Takeishi ${ }^{a}$ for The Telescope Array Collaboration \\ ${ }^{a}$ Institute for Cosmic Ray Research, University of Tokyo, Kashiwa, Chiba, Japan \\ ${ }^{b}$ High Energy Astrophysics Institute and Department of Physics and Astronomy, University of \\ Utah, Salt Lake City, Utah, USA \\ e Department of Physical Sciences, Ritsumeikan University, Kusatsu, Shiga, Japan \\ ${ }^{f}$ Graduate School of Science, Osaka City University, Osaka, Osaka, Japan \\ E-mail: nonaka@icrr.u-tokyo.ac.jp
}

The Telescope Array (TA) experiment, located in the western desert of Utah, USA, at $39.38^{\circ}$ north and $112.9^{\circ}$ west, is collecting data of ultra high energy cosmic rays in the energy range $10^{18}$ - $10^{20} \mathrm{eV}$. The experiment has a Surface Detector (SD) array surrounded by three Fluorescence Detectors (FD) to enable simultaneous detection of shower particles and fluorescence photons. Measurement of shower particles at ground level, with different absorber thickness, enables a more detailed study of the experiment's energy scale and of hadron interaction models. In this report, we present a design and first observation result of a surface muon detector using lead plate and concrete absorbers.

The 34th International Cosmic Ray Conference,

30 July- 6 August, 2015

The Hague, The Netherlands

${ }^{*}$ Speaker. 


\section{Introduction}

The Telescope Array (TA) experiment was designed to have both the fluorescence detectors (FD) and a surface detector (SD) array using plastic scintillators. Totally 507 SDs are deployed with $1.2 \mathrm{~km}$ separation. Three FD stations are built on the perimeter of the SD array. From the observations with fluorescence telescopes, energies are obtained from observed air shower cascade developments. For air showers detected with the surface detector, the energy of primary cosmic ray is estimated by comparing the measured energy deposit at the core distance of $800 \mathrm{~m}$ with that from Monte Carlo (MC) simulations. Using hybrid events which FD also measures calorimetric energy, it is known that there is a systematic difference between the energy that measured with the SD array and that with the FDs. The correction factor to match with FD energy is -27\% [1]. This means that the estimated energy deposit at surface detectors with MC simulation is smaller than the real. There are reports of enhanced muon flux in the lateral distribution of shower particle also [2]. The muon component might be a part of reason for the discrepancy of energy scale by FD and SD in TA experiment. Our idea is that a precise measurements of the air shower muons will give information to show what aspect of MC is different from observed air showers. The detailed measurement of the shower at this energy region will give the unique information about MC simulation especially at the energy region between $10^{18.7} \mathrm{eV}$ to $10^{19.0} \mathrm{eV}$, where the chemical composition is quite consistent with proton dominated composition. This report is about the surface detectors which were developed for that purpose and their operational status.

\section{Concept of detector}

It is planned to have multiple types of detectors with different thicknesses of absorber, to measure shower particles details of such as arrival timing and energy distribution. One type is the lead inserted segmented detector, which will have the capability to measure muon and electro magnetic (EM) components separately. And second type is a concrete shielded detector to find high energy $(E>600 \mathrm{MeV})$ muons.

\subsection{Lead inserted scintillator (Lead Burger)}

The lead burger type of detector was previously used as an associated measurement in the AGASA experiment[3, 4]. Fig. 1 shows a schematic of side view of the concept of the detector in TA. The detector has two layer of scintillator with $12 \mathrm{~mm}$ thickness and lead plates are inserted between scintillators to have the thickness of $25 \mathrm{~mm}$. Scintillation light is collected through WLS fibers layed in grooves in the surface of scintillator with $20 \mathrm{~mm}$ spacing. While most of electrons are absorbed in the lead plate, most of muons can penetrate all layers of the detector and leave signals in both top and bottom layer. Gamma rays which produce secondary electrons leave signals only in the bottom layer. Hence, it gives information of number of muons. Energy deposit from electromagnetic component also can be measured with top layer. While counting the number of muons, the accuracy of measurement depends not only on the thickness of lead but also size of detector segment and time constant of the front end electronics. The current detector is segmented into $0.75 \mathrm{~m}^{2}$ of surface each. Changing the time constant of electronics and segment size has almost same effect on accuracy of counting of muons. As an optimum time constant and segment 
size we set value of time constant as $1 / 3$ of the existing TA SD electronics. Fig. 1 shows schematic side view of the detector.

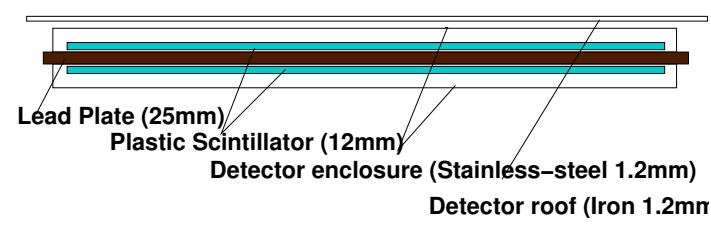

Figure 1: A side view of detector showing majour component of lead inserted detector.

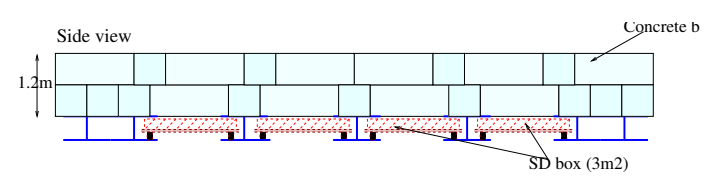

Figure 2: A side view of shielded muon detector. Below the shield, there are 4 slots which each hold two scintilltor units. Total surface of sensitive part is $24 \mathrm{~m}^{2}$.

\subsection{Concrete shield detector}

The concreate shield detector is designed to have $1.2 \mathrm{~m}$ of absorber. Concrete absorber was assembled from concrete blocks. Each of dimension is $60 \times 60 \times 140 \mathrm{~cm}^{3}$. It is removable with crane if necessary. Eight scintillator segment units are stationed under the concrete absorber. Each unit has $3 \mathrm{~m}^{2}$ of surface area. The scitillator unit is the same with the one which is used as TA SD [5]. Each unit has two layers of scintillator, each $1.2 \mathrm{~cm}$ thick. Signals are sampled and digitized with $50 \mathrm{MHz}$ FADC. The sampling rate is the same in the lead detector. We can also estimate the muon number by using the coincidence rising edge in wave form. Since the concrete absorber thickness is $1.2 \mathrm{~m}$, detected particles are purely muons except for the events with shower core are located very near the detector. In the event that the number of incident particles is large, it is still possible to count number of hit particles using charge output. Fig. 2 shows the schematic side view of the concrete based detector.

\section{Detector simulation}

Simulations of hitting to the detector are generated from a shower data base and, and GEANT4 simulation [6] is used for particle's energy deposit and timing. From the simulated energy deposit in each time bin, the energy deposit is converted to charge amount by adding the fluctuation of output photo electrons. It is known that the average number of photo-electrons $\left(N_{p e}\right)$ for $2.4 \mathrm{MeV}$ of energy deposit gives around 20 pe on average in our detector. The integrated FADC out put for the $1 \mathrm{mip}$ signal is tuned to be around 50 counts. We consider this condition as the standard detector operating mode while simulating energy deposit from GEANT4. To simulate the wave form, the front end electronics is also considered when forming the wave form trace. A charge output from a short pulse from a PMT is shaped with a function $F(t)=B \times t^{s}\left(\frac{e^{-\frac{a t}{2}}+e^{-\frac{b t}{2}}}{b-a}\right)^{2}$ where $t$ is time $(n s)$ from energy deposit. The constants ( $s, B, a$ and $b)$ are typical value of TA SD [7]. With the time constants of front-end electronics, a signal from a single muon gives asymmetric shaped pulse with 12 counts in height and $\sim 320 \mathrm{nsec}$ of FADC trace. For this measurement, we plan to optimize the time constant to obtain better accuracy for muon counting. In the simulation, time constant was set to be $1 / 3$ of the normal TA SD resultng in a typical pulse length of about $120 \mathrm{nsec}$. 


\subsection{Lead inserted detector}

At distances greater than $1200 \mathrm{~m}$ from the core, the number of electro-magnetic(EM) particles above $50 \mathrm{MeV}$ are greatly reduced, while most muons still have high energy. It is expected that at these distances, the lead burger detectors will get a signal only in the upper scintillator from electrons. Gamma rays at this energy will leave a signal only in the lower scintillator and muons will leave signals in both layers of scintillator. There may still be some penetrating high energy EM component, but it is expected to be small. If a waveform rises at $\geq 3$ count/bin, then this was defined as the rising edge. If the rising edge is coincident within $\pm 20 \mathrm{nsec}$ between top and bottom layer, this is counted as arrival of one muon. In the case the number of particles becomes larger, it becomes difficult to pick up rising edge from a single particle. Then miss-count of the muon numbers increases. Fig. 3 shows lateral distribution of simulated muon from vertical proton shower $\left(10^{19.0} \mathrm{eV}\right)$. The $\star$ represents the number of thrown muons. The $\mathbf{r e p r e s e n t s ~ t h e ~ c o u n t e d ~}$ number of coincidences in the rising edge of the wave form. The $\square$ represents the number of thrown muons counted via coincidence of the rising edge. The EM component is also injected while the simulation. Fig. 4 shows fraction of counted muon number to thrown number of muon as a function of core distance. The $\boldsymbol{\square}$ is the fraction of counted number of coincidences. The $\square$ is the fraction of thrown muon counted coincidences. When the core distance is $\geq 1200 \mathrm{~m}$, the detector and counting method gives $20 \%$ of accuracy in the average number of muons. This same

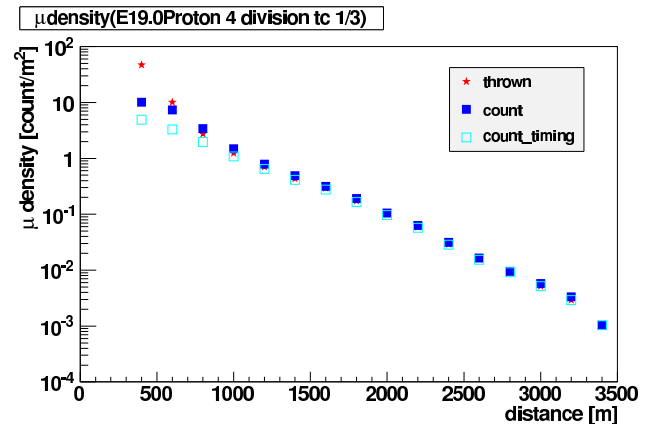

Figure 3: Average number of thrown muon from $10^{19} \mathrm{eV}$ air shower and counted muon. $\star$ : number of thrown muons. $\mathbf{\square}$ : counted number of coincidences. $\square$ : number of thrown muons counted with coincidence

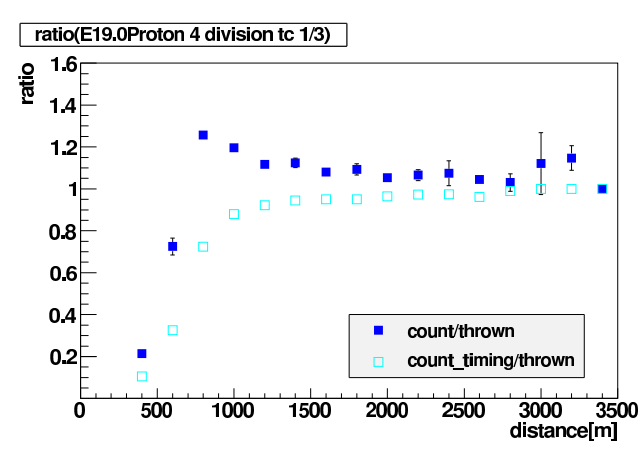

Figure 4: Fraction of the counted number of muons to thrown number.

simulation was perfomed while varying the energy of the primary particle. Table. 1 shows estimated minimum distance from the core which gives an accuracy of $\sim 20 \%$ for the measured number of muons.

\section{2 concrete detector}

Fig. 5 shows the energy distribution of muons at the top of the concrete shield and for muons which penetrate the concrete shield. For $1.2 \mathrm{~m}$ of concrete shield $\sim 600 \mathrm{MeV}$ is the threshold energy. The fraction of the high energy muon to all arrival muons is plotted in Fig. 6. At core distance $\geq 1200 \mathrm{~m}$, the counted number of muons is about 0.6 times smaller than that at surface of the concrete detector. There is dependence on the core distance also. Fig. 7 shows simulation of 


\begin{tabular}{|l|c|}
\hline Primary Energy $(\mathrm{eV})$ & distance $(\mathrm{m})$ \\
\hline $10^{18.0}$ & $>1000$ \\
\hline $10^{18.4}$ & $>1000$ \\
\hline $10^{18.7}$ & $>1200$ \\
\hline $10^{19.0}$ & $>1200$ \\
\hline $10^{19.4}$ & $>1400$ \\
\hline
\end{tabular}

Table 1: Estimated distance from the core for which the lead muon detector can measure number of muons with about $20 \%$ of accuracy greater than $80 \%$ purity in the muon fraction.

accuracy of muon counting for the concrete detector. A scatter plot shows the counted number of muons and number of muons just under the concrete shield. Two methods of counting were tried. One method used the coincidence of the rising edge between top and bottom layers. The scatter plot is displayed in the left side of figure. It shows better resolution at small number of incident particles as compared to the plot displayed at the right side. The right side plot shows the counted number of muons based on observed charge divided by VEM in the lower layer of scintillator.

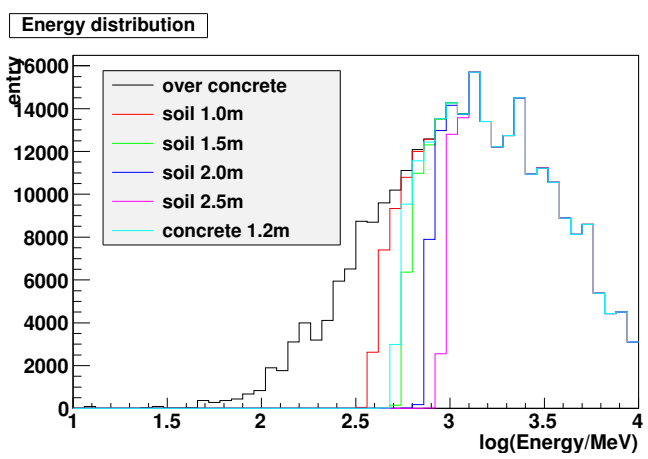

Figure 5: Absorber thickness and detectable muon energy.

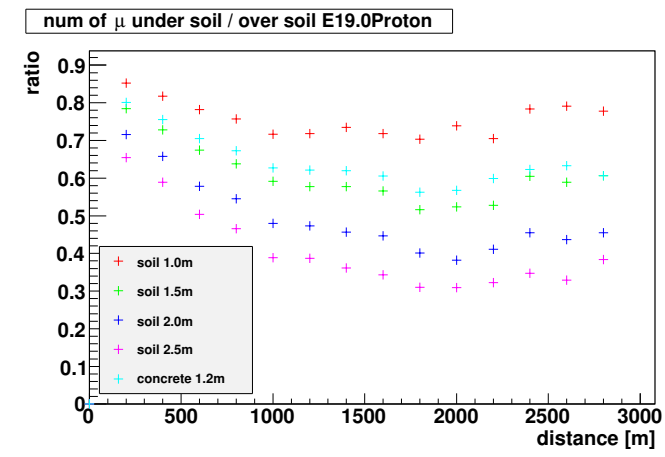

Figure 6: Ratio of muons which penetrate the concrete absorber to reach the counter.
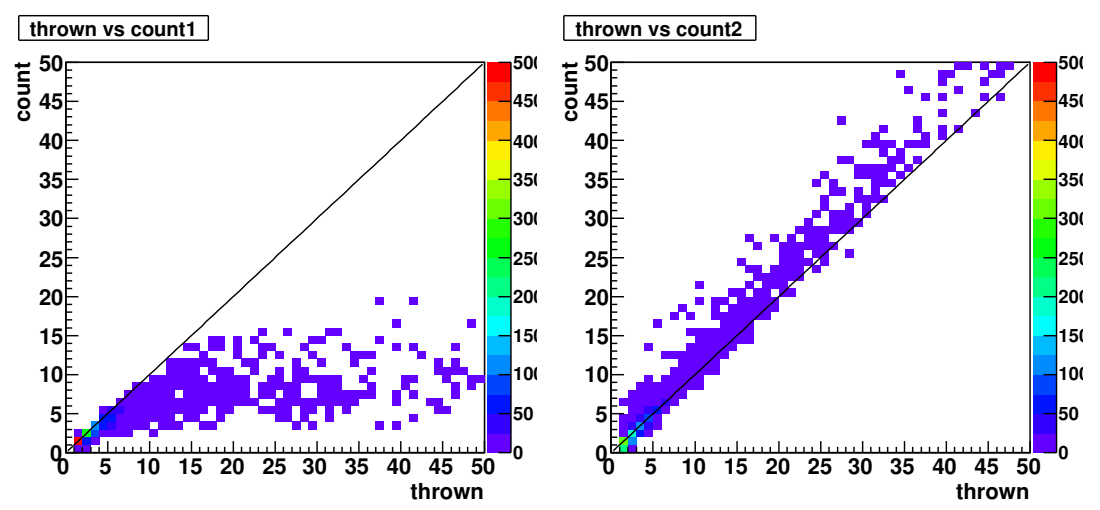

Figure 7: Scatter plot of the thrown and counted number of muons (left:using waveform right:using charge). 


\section{Construction and prototype observation}

\subsection{Detector assembly and performance}

For the concrete shield detector, a total of 8 modules of $3 \mathrm{~m}^{2}$ TA SD are assembled. For the lead detector, 12 modules (top and bottom pair) are assembled, each of $0.75 \mathrm{~m}^{2}$. Fig. 8 shows the integrated FADC count for a minimum ionising particle (1mip). The $N_{p e}$ for $1 \mathrm{mip}$ was 18.3 pe. The average $N_{p e}$ for $1 \mathrm{mip}$ at TA SD is $24.6 \pm 7.2$ with same type of PMTs. Fig. 9 shows position dependence of the response for the mip. The measurement of response was done at 35 positions to cover entire segment which is $15 x 15 \mathrm{~cm}^{2}$. The RMS of the distribution of $1 \mathrm{mip}$ value is about $5 \%$ of the average $1 \mathrm{mip}$ value.

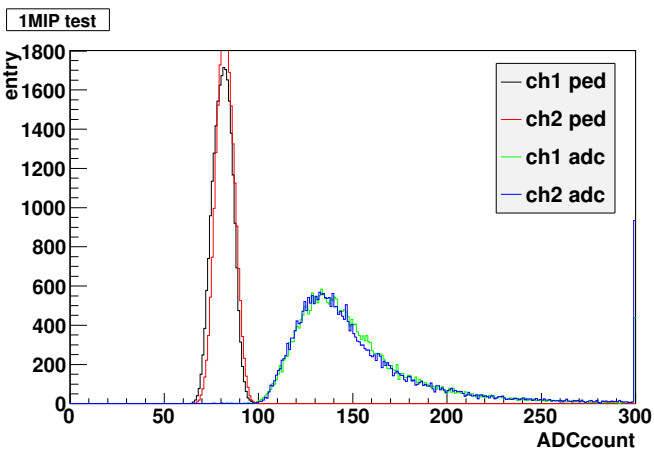

Figure 8: The integrated FADC count for a minimum ionising particle (1mip).

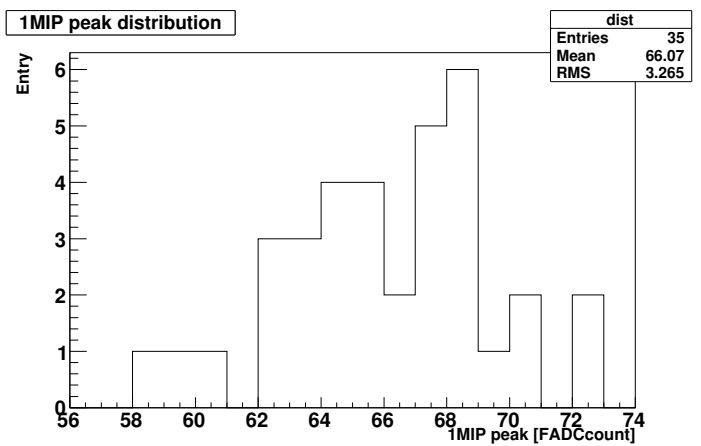

Figure 9: position dependence of the response for the mip.

\subsection{Test observation of air shower}

We started test observation with a prototype lead detector in December 2013. Fig. 10 shows comparison with count rate of the first lead inserted detector and its variation for one month. It is seen that count rate variation and ratio with other surrounding detectors are very stable. Fig. 11 shows the distribution of energies of observed air showers. The black histogram shows all reconstructed showers. The green shows the reconstructed events with core position within $3 \mathrm{~km}$ of the lead detector. The red histogram shows the event with core position within $3 \mathrm{~km}$ and for which the lead detector recorded the wave form. Of the 108 showers detected less than $3 \mathrm{~km}$ away, 18 events had upper and lower coincident signals. The comparison of detected signal and MC is under way. Fig. 12 shows one of observed events in this time period. The reconstructed energy is $17 \mathrm{EeV}$ and zenith angle is $37^{\circ}$. The recorded VEM at top layer of the prototype detector is consistent with the other detector. Fig. 13 shows signal timing at each detector displayed with lead detectors timing. The horizontal axis is the depth along the shower axis calculated with the reconstructed shower geometry. Timing is also well aligned along other detectors. So recorded waveform at the lead detector can be considered as a signal associated with this shower. Fig. 14 shows the wave form recorded at lead detector for this event.

\section{Summary and current status}

To study the detail of discrepancy between MC and observed showers, we designed lead in- 


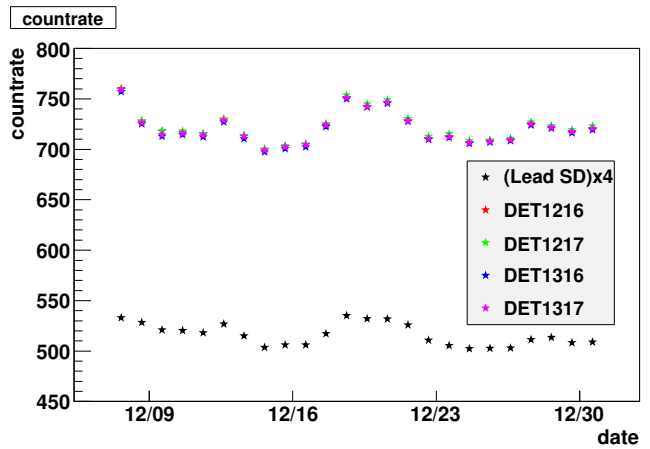

Figure 10: Variation of count rate in one month of operation.

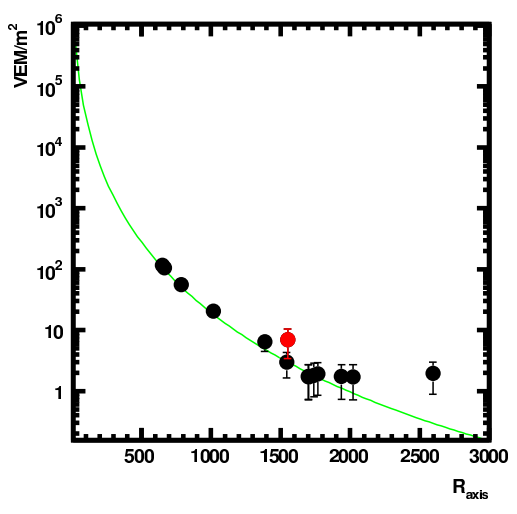

Figure 12: An example of lateral distribution at observed event and comparison with the lead detector. The black points show data point from standard TA SD. The green line is fitted Lateral Distribution Function. Signal of top layer of the lead detector is displayed in red marker.

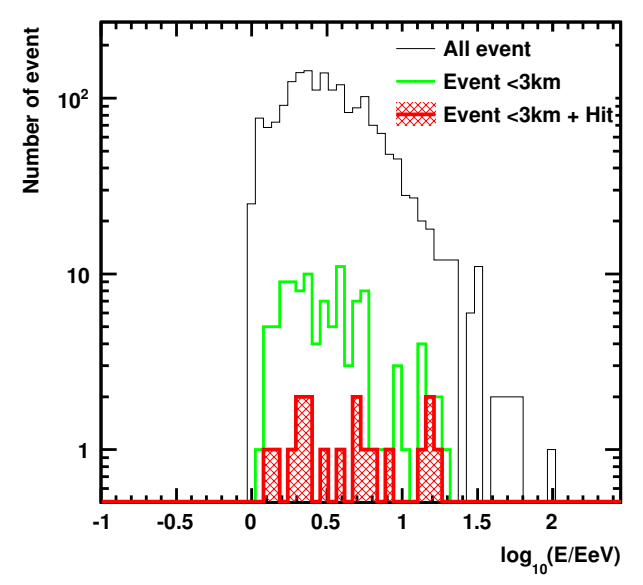

Figure 11: Energy distribution of observed event in six months of operation. Green histogram shows energy distribution of the shower detected within $3 \mathrm{~km}$ of distance from detector. Red is event lead detector had signal associate with the shower.

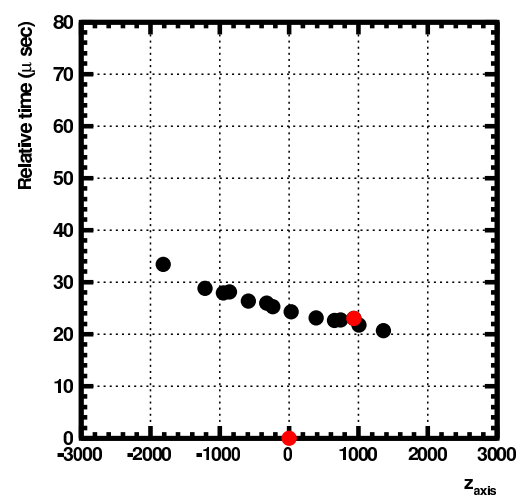

Figure 13: Timing comparison at same event.

serted detectors and concrete shield detectors operated as a part of the TA SD. From the simulation, we studied the expected accuracy of muon counting at various energies and core distances. The lead detector is expected to provide a good measurement of muon number together with electromagnetic component relatively far from shower core $(\geq 1200 \mathrm{~m})$ with the current design. The concrete shielded detector is expected to provide good muon number resolution for wide range of incident particles due to FADC sampling and the segmentation of detector. All of detectors have 

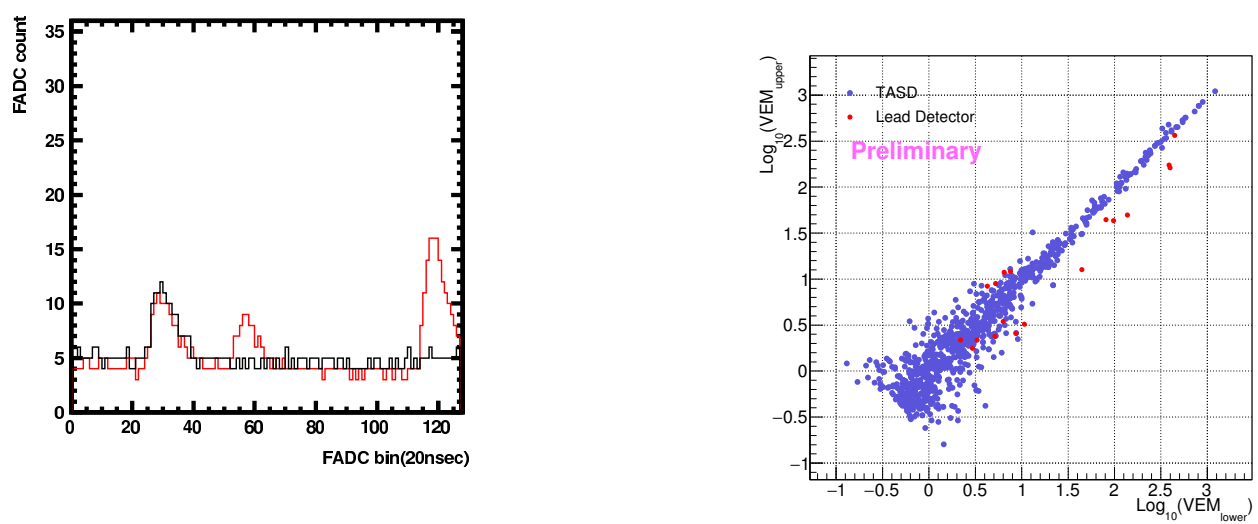

Figure 14: Observed wave form at the lead detector for the same event. The black line shows the wave form of the lower layer and the red shows that the top Figure 15: Charge ratio between signals from upper and lower layer. layer.

been assembled in Japan and have been shipped to the TA site. The detectors are currently being installed for the test observations in the TA array. The detector has good stability and sensitivity and uniformity which is expected from TA SD. The detector collects the data of wir showers in association with the TASD. With more statistics, detailed comparison with existing MC will be done. Construction of concrete absorber also started at June 2014 and finished March 2015. Currently 8 of $3 \mathrm{~m}^{2}$ detector segment are being installed.

\section{Acknowledgements}

The Telescope Array collaborators and acknowledgement is listed in the URL in foot note. ${ }^{1}$.

\section{References}

[1] T.Abuzayad,et.al ApJ 768 L1 (2013)

[2] R.Engel, et.al Proceedings of the 30th ICRC in Merida,Vol 4 385,(2007)

[3] N. Chiba et.al, Nuclear Instrumnment and Method in Physiscs Research A ,311,1-2,338 (1992)

[4] K. Honda et.al Phys.Rev.D. 56,3833,(1997)

[5] T.Abuzayad, et.al Nuclear Instrunment and Method in Physics Research A 689,87-97 (2012)

[6] S.Agostinelliae,et.al Nuclear Instruments and Methods in Physics Research A 506,250âĂŞ303,(2003)

[7] A. Taketa, et al.Doctor Thesis, University of Tokyo, (2012)

\footnotetext{
${ }^{1}$ http://www.telescopearray.org/images/papers/ICRC2015-authorlist.pdf
} 